\title{
ESTUDO DE VIABILIDADE ECONÔMICA PRELIMINAR PARA A PRODUÇÃO DE BIODIESEL POR DESTILAÇÃO REATIVA
}

\author{
J. P. SILVA ${ }^{1}$, T. P. C. SOUZA ${ }^{1}$ D. E. S. CAVALCANTI ${ }^{1}$ B. F. SANTOS ${ }^{1}$ E J. M. F. SILVA ${ }^{1}$ \\ ${ }^{1}$ Universidade Federal de Pernambuco, Departamento de Engenharia Química \\ E-mail para contato: josivan_silva@hotmail.com
}

\begin{abstract}
RESUMO - O biodiesel é um combustível renovável, cujo a demanda por tem crescido nos últimos tempos, o processo de produção por destilação reativa tem se mostrado mais favorável que o processo convencional de produção. Diversos trabalhos na literatura demonstram as vantagens significativas da destilação reativa sobre o processo sequencial convencional, como por exemplo, a alta taxa de conversão química e o custo reduzido de operação. Este estudo teve como objetivo analisar a viabilidade econômica preliminar da produção de biodiesel utilizando a destilação reativa, isto foi feito partindo de condições otimizadas de produção, e seguindo o método do Custo Anualizado Total Unitário (CATU), que leva em consideração o custo fixo total anualizado do investimento assim como os lucros obtidos. Partindo deste estudo foi possível concluir que a destilação reativa se mostra promissora quando se deseja reduzir custos e aumentar a produtividade de biodiesel.
\end{abstract}

\section{INTRODUÇÃO}

O biodiesel é um combustível renovável, que pode ser utilizado como um substituto do diesel, o biodiesel pode ser produzido por transesterificação de um óleo ou gordura, junto a um álcool como metanol ou etanol, sendo metanol o mais utilizado. A demanda por biodiesel tem crescido nos últimos tempos de acordo Beatriz et al. (2011), e a destilação reativa tem se mostrado economicamente mais favorável que o processo convencional de produção, pois é uma operação química que reúne em uma unidade reações químicas e separação em um mesmo processo, a destilação reativa se torna uma alternativa efetiva para a combinação tradicional de reatores e separadores especialmente quando se trata de reações reversíveis, como a transesterificação de óleos vegetais. Muitas vezes os custos associados à produção de biodiesel são elevados, tornando pouco viável a produção deste biocombustível, um estudo de viabilidade econômica se mostra necessário, a partir do mesmo é possível verificar se o processo pode mesmo ser posto em prática, e gerar lucros para o investidor.

O estudo da viabilidade econômica pode ser feito seguindo o método do Custo Anualizado Total Unitário CATU (Santana et al., 2010; Haas, et al. 2006). O cálculo leva em consideração o custo fixo total anualizado do investimento com os custos variáveis de processo, todos atrelados à produção anual (capacidade da planta). Este mesmo método de análise econômica foi usado para comparar os efeitos da operação do processamento de Gás Natural em Plataforma Off-Shore (Sant'Anna, 2005). 
Segundo Douglas (1988) e Hapel e Jordan (1975) o custo fixo anualizado (US\$/ano) corresponde aos custos associados ao investimento nos equipamentos centrais (ISBL - Inside Battery Limits equipamentos) mais o custo operacional anual (ISBL Inside Battery Limits - operacional) que corresponde ao total gasto em utilidades (energia) e insumos (matérias-primas). O cálculo do CATU pode ser feito levando em consideração a soma do custo fixo com o custo operacional dividido pelo valor da produção (US\$/ano) como descrito pela Equação 1.

$$
C A T U=\frac{I S B L(\text { equip })+I S B L(\text { oper })}{\text { Produção Anual }}
$$

Cada equipamento no processo possui um custo de projeto (ISBL) que será somado a fim de se obter o custo total dos equipamentos usados no processo. O cálculo dos ISBL dos equipamentos pode ser feito somando-se o preço de compra dos equipamentos, conforme Equação 11, multiplicando-se o somatório por um fator experimental. No presente trabalho, foi utilizado o fator de Lang (fL), pois o mesmo é a estimativa simplificada em função do tipo de processamento.

$$
\operatorname{ISBL}(\text { equip })=f_{L} \sum I_{E i}
$$

O valor para o fator de Lang depende do tipo de processo usado, e o mesmo pode ser obtido pelo uso das Tabelas 1 e 2, e o valor fL pode ser calculado pela Equação 3 apresentada a seguir.

$f_{L}=\left(1+f_{A}\right)\left(1+f_{B}\right)$

fA e fB podem são obtidos pelas Equações 4 e 5 abaixo.

$$
f_{A}=\sum_{1}^{9} f_{i}
$$

$f_{B}=\sum_{10}^{12} f_{i}$

Os valores das constantes para o ISBL de cada equipamento dependem do projeto, sendo corrigidos periodicamente pelo índice Marshall \& Swift (http://www.che.com/).

No caso da destilação reativa, será levado em consideração a coluna de destilação e seus internos, assim como também em consideração o custo de operação e projeto. Os valores calculados para o custo do processo serão divididos pelo valor da produção anual, obtendo-se assim, o custo anualizado total unitário CATU. Já o valor da viabilidade econômica preliminar poderá ser obtido da seguinte relação (Equação 6) dada a seguir:

Viabilidade Econômica $=(1-C A T U) \cdot 100$ 
Tabela 1 - Valor para o fator de Lang A

\begin{tabular}{lll}
\hline Fator & Tipo & Valor \\
\hline f1 & Instalação & 0,15 \\
$\mathrm{f} 2$ & Isolamento & 0,15 \\
$\mathrm{f} 3$ & Tubulação & 0,75 \\
$\mathrm{f} 4$ & Fundações & 0,10 \\
$\mathrm{f5}$ & Edificações & 0,07 \\
$\mathrm{f6}$ & Estruturas & 0,06 \\
$\mathrm{f} 7$ & Prevenção de incêndios & 0,06 \\
$\mathrm{f} 8$ & Instalações elétricas & 0,10 \\
$\mathrm{f} 9$ & Pintura e limpeza & 0,06 \\
\hline
\end{tabular}

Tabela 2 - Valor para o fator de Lang B

\begin{tabular}{lll}
\hline Fator & Tipo & Valor \\
\hline $\mathrm{f} 10$ & "Overhead" e custo de montagem & 0,30 \\
$\mathrm{f} 11$ & Serviços de engenharia & 0,13 \\
$\mathrm{f} 12$ & Eventuais & 0,13 \\
\hline
\end{tabular}

\section{METODOLOGIA}

O estudo da viabilidade econômica pode ser feito seguindo o método do Custo Anualizado Total Unitário CATU (Santana et al., 2010; Haas, et al. 2006). O cálculo é feito a partir das Equações 1 a 6, e resulta em um valor que define a viabilidade do investimento, para valores negativos, há prejuízo e o investimento leva a uma despesa maior que o lucro resultante, para viabilidade igual a zero não há lucro ou prejuízo, para valores superiores a zero há lucro, o lucro é tão maior quanto for o valor da viabilidade econômica preliminar.

Para o processo de produção e biodiesel é necessário considerar, ainda, diversos equipamentos, tais como decantadores, tanques e armazenamento entre outros, o fluxograma sugerido que foi avaliado economicamente está exposto na Figura 1 


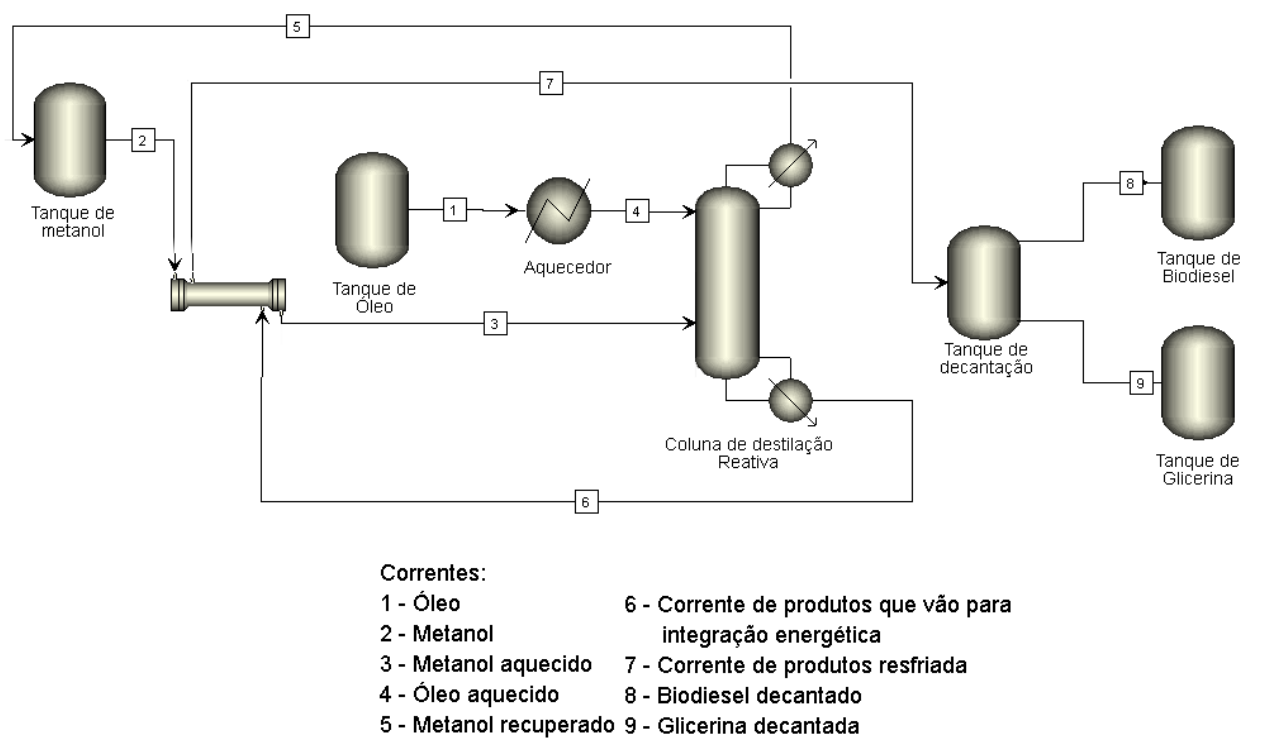

Figura 1: Fluxograma sugerido para a avaliação econômica preliminar.

A Figura 1 expõe um fluxograma simplificado para produção de biodiesel utilizando destilação reativa, onde o metanol e o óleo necessitam ser aquecidos para entrar na coluna com as condições adequadas, a carga térmica para aquecimento do metanol é proveniente de integração energética, sendo que as condições de temperatura impedem que o mesmo possa ser feito com o óleo, por isso o mesmo precisar ser direcionado a um aquecedor, com as correntes 3 e 4 nas condições adequadas, a coluna de destilação reativa processa a conversão em biodiesel, gerando também glicerina como subproduto, a corrente de topo consiste de metanol puro, e pode ser recuperado, já a corrente que contém os produtos, como descrito, é direcionada a um trocador de calor para aquecimento do metanol de alimentação, em seguida segue para um decantador, onde o biodiesel e a glicerina são separados.

Um algoritmo foi desenvolvido em MATLAB ${ }^{\circledR}$ para cálculo da viabilidade econômica, e segue a rotina exposta por Perlingeiro (2011). O cálculo foi feito para uma jornada diária de 8 horas, 5 dias por semana e 52 semanas por ano. No estudo realizado o valor do biodiesel foi obtido a partir do $24^{\circ}$ leilão ANP. O preço utilizado nos cálculos dos compostos utilizados no processo estão expostos na Tabela 3.

Tabela 3 - Valores de insumos e produtos na produção de biodiesel

\begin{tabular}{c|c}
\hline Composto & Preço médio \\
\hline Metanol & $1,70 \mathrm{R} \$ / 1$ \\
\hline Óleo vegetal & $1,70 \mathrm{R} \$ / 1$ \\
\hline Hidróxido de sódio & $1,00 \mathrm{R} \$ / \mathrm{Kg}$ \\
\hline Biodiesel & $2,396 \mathrm{R} \$ / 1$ \\
\hline Glicerina & $0,50 \mathrm{R} \$ / 1$ \\
\hline
\end{tabular}


Foi considerado o preço do dólar como R \$ 2,2357 em 19/04/2014, o estudo foi feito para a segunda condição otimizada da dissertação de Silva (2013), pois apresentou uma configuração onde os equipamentos tem menores dimensões e perda de carga, assim como uma utilização de metanol inferior a primeira condição otimizada, nesta condição se utiliza $100 \mathrm{~kg}$ de trioleína (óleo) por hora, assim como $0,5 \mathrm{Kg} / \mathrm{h}$ de hidróxido de sódio e 10,87 Kg/h de metanol reagem e $1,42 \mathrm{~kg} / \mathrm{h}$ saem com a corrente de fundo, totalizando $12,29 \mathrm{~kg} / \mathrm{h}$ de metanol sendo consumido.

Para cálculo do CATU é necessário se utilizar o índice Marshall e Swift, que no $1^{\circ}$ trimestre de 2014 tem valor estimado de 1843,8 segundo a revista chemical engineering. Foram levadas em consideração as dimensões resultantes do dimensionamento realizado por Silva (2013), utilizando o Aspen Plus ${ }^{\circledR}$ para as dimensões que garantiam o tempo de residência necessário. Considerou-se a coluna como sendo de aço inox, os cálculos também consideram custos com refervedor, condensador, decantador, tanques de armazenamento e bombas, Sendo a coluna de destilação e o decantador com $0,11 \mathrm{~m}^{3}$ de volume cada, e os 4 tanques de armazenamento com $1,57 \mathrm{~m}^{3}$ cada. $\mathrm{O}$ custo com energia elétrica tanto para aquecimento quanto para as bombas também foram levados em consideração, tendo como referência o custo do KWh informado pela ELETROACRE (Companhia Energética do Acre) de R\$ 0,42 KWh em 19/04/2014 pois este é o KWh mais caro do Brasil, utilizando assim o caso mais pessimista possível. O cálculo também leva em consideração o salário associado a um operário trabalhando na operação do equipamento.

\section{RESULTADOS E DISCUSSÃO}

O programa desenvolvido em MATLAB $^{\circledR}$ utiliza as equações de 1 a 6 pelo processo descrito na metodologia. Gerando as seguintes informações:

\section{AVALIAÇÃO ECONÔMICA PRELIMINAR}

\section{PRIMEIRO ANO:}

Valor CATU: 1.086

Viabilidade econômica: -8.59

Investimento total: US\$96446.80

Custo total com equipamentos: US\$ 24729.95

Custo com coluna: US\$ 1648.55

Custo com refervedor: US\$ 50.83

Custo com condensador: US\$130.51

Custo com decantador: US\$ 1260.53

Custo com tanques de armazenamento: US\$ 20777.77

Custo com bombas: US\$ 861.76

Custo anual com energia: US\$ 3323.85

Custo anual com insumos: US\$ 195135.52

Lucro líquido anual: US\$ -24296.07

Lucro líquido anual: R\$ -54318.73 


\section{APÓS O PROCESSO PAGO}

Valor CATU: 0.7448

Valor da viabilidade econômica: 25.52

Custo anual com energia: US\$3323.85

Custo anual com insumos: US\$ 195135.52

Lucro líquido anual: US\$ 72150.73

Lucro líquido anual: $\mathrm{R} \$ 161307.39$

Os resultados apresentados acima mostram que o processo não é economicamente viável no primeiro ano de operação, pois a viabilidade econômica no primeiro ano é de $-8,59 \%$, entretanto a viabilidade econômica sobe para $25,52 \%$ do segundo ano em diante, mostrando que o processo é economicamente viável do segundo ano em diante. O investimento inicial é de US\$ 96446,48 equivalente a $\mathrm{R} \$ 215630,00$, onde se inclui custo com equipamentos e as correções referentes ao fator de Lang, levando a um lucro líquido anual de US\$ 72150,73 ou equivalente a R \$161307,39 no segundo ano, o que compensa o déficit no primeiro ano de $\mathrm{R} \$ 54318,73$, sendo isto para se obter uma conversão em biodiesel acima de 99,9\%.

O trabalho de Silva (2013) resulta em uma configuração final na qual se atende a necessidade de purificação dos produtos e conversão necessária para adequar o biodiesel as normas da ANP (Agência nacional do petróleo e biocombustíveis), pois foram reduzidos o tempo de residência e as dimensões da coluna modelada. Perante esta condição mais favorável uma nova avaliação econômica preliminar foi realizada:

\section{AVALIAÇÃO ECONÔMICA PRELIMINAR}

\section{PRIMEIRO ANO:}

Valor CATU: 0.9539

Viabilidade econômica: 4.61

Investimento total: US\$ 95940.05

Custo total com equipamentos: US\$24600.01

Custo com coluna: US\$1518.61

Custo com refervedor: US\$50.83

Custo com condensador: US $\$ 130.51$

Custo com decantador: US\$ 1260.53

Custo com tanques de armazenamento: US\$20777.77

Custo com bombas: US $\$ 861.76$

Custo anual com energia: US\$ 3776.71

Custo anual com insumos: US\$292703.27

Lucro líquido anual: US\$19541.63

Lucro líquido anual: $\mathrm{R} \$ 43689.22$ 


\section{APÓS O PROCESSO PAGO}

Valor CATU: 0.7277

Valor da viabilidade econômica: 27.23

Custo anual com energia: US\$ 3776.71

Custo anual com insumos: US\$292703.27

Lucro líquido anual: US\$ 115481.68

Lucro líquido anual: $\mathrm{R} \$ 258182.39$

Como é possível observar as condições de operação no configuração final, tem diversas vantagens sobre a condição avaliada anteriormente. Pois apresenta uma condição de menor tempo de residência e maior produtividade, assim é possível produzir mais no mesmo período de tempo (Silva, 2013). O tempo de residência menor reflete no custo de investimento e operação, onde o estudo de viabilidade econômica preliminar, mostra um lucro de $\mathrm{R} \$ 43689,22$ no primeiro ano de operação, quando na verdade se observou na condição anterior um déficit de $\mathrm{R} \$-54318,73$. A redução do tempo de residência foi obtida pela diminuição do número de pratos, o que levou a uma coluna menor e consequentemente mais barata, custando US\$1518,61, em contrapartida a coluna da condição anterior custou US\$ 1648,5, mostrando uma redução de custo e maximização de lucros efetiva.

\section{CONCLUSÕES}

$\mathrm{O}$ estudo de viabilidade econômica deste trabalho demonstra que a coluna resultante do segundo planejamento fatorial pode levar a um lucro líquido de $\mathrm{R} \$ \mathrm{R} \$ 161307,39$ anual após o segundo ano de operação, mesmo sendo uma coluna com dimensões compatíveis com as de uma planta piloto. A coluna resultante do segundo planejamento fatorial mostra uma viabilidade econômica de $-8,59$ \% no primeiro ano, com déficit de $\mathrm{R} \$-54318,73$, sendo pago no segundo ano de operação com um valor da viabilidade econômica de $25,52 \%$, e um lucro líquido anual de $\mathrm{R} \$$ 161307,39, mostrando que o processo em questão é rentável, mesmo em pequena escala. As condições resultantes do processo de otimização do trabalho de Silva (2013) levam a uma coluna que gera $\mathrm{R} \$ 43689,22$ como lucro anual no primeiro ano e $\mathrm{R} \$ 258182,39$ do segundo ano em diante, mostrando uma viabilidade econômica maior. Pode-se concluir que o processo de destilação reativa para a produção de biodiesel é viável e economicamente sustentável.

\section{REFERENCIAS}

BEATRIZ A.; ARAÚJO Y. J. K.; LIMA D. P.; Glycerol: a brief history and their application in stereoselective syntheses. Quím. Nova vol.34 no.2 São Paulo 2011

DOUGLAS, J.M. Conceptual design of chemical process. McGraw-Hill, New York (1987). 1988 
HAAS M. J.; MCALOON A. J.; YEE W. C.; FOGLIA T. A. A process model to estimate biodiesel production costs. Bioresource technology, V97, pp 671 - 678, 2006.

HAPPEL, J.; JORDAN, D.G. Chemical process economics. Chapman and Hall, New York, NY. 1958. - 289 p.

PERLINGEIRO, C. A. G. Engenharia de processos, análise, simulação, otimização e síntese de processos químicos, Blusher, Rio de Janeiro, pp 77-90, 2011.

SANT'ANNA A. A.; MEDEIROS J. L.; ARAÚJO O. Q F. Simulação de processamento de gás natural em plataforma off- shore. $3^{\circ}$ Congresso Brasileiro de P\&D em Petróleo e Gás, 2005.

SANTANA, G. C. S.; MARTINS, P. F.; DA SILVA, N.; BATISTELlA, C. B.; FILHO, R. M.; MACIEL, M. R.W. Simulation and cost estimate for biodiesel production using castor oil. Chemical engineering research \& design, V88, pp 626 - 632, 2010.

SILVA J. P. Avaliação por planejamento fatorial da produção de biodiesel via destilação reativa Dissertação (Mestrado em Engenharia Química), Universidade Federal de Pernambuco, UFPE. Recife, Pernambuco, 2013. 